Cahiers de recherches médiévales

\title{
Christine de Pizan et la répétition de l'histoire
}

Exempla historiques et discours à caractère prophétique dans le livre I du Livre de l'advision Christine

\section{Claire Le Ninan}

\section{(2) OpenEdition}

\section{Journals}

Édition électronique

URL : https://journals.openedition.org/crm/5822

DOI : $10.4000 / \mathrm{crm} .5822$

ISSN : $1955-2424$

Éditeur

Honoré Champion

Édition imprimée

Date de publication : 20 juin 2008

Pagination : 239-251

ISSN : $1272-9752$

\section{Référence électronique}

Claire Le Ninan, "Christine de Pizan et la répétition de l'histoire », Cahiers de recherches médiévales [En ligne], 15 | 2008, mis en ligne le 20 juin 2011, consulté le 15 décembre 2022. URL : http:// journals.openedition.org/crm/5822 ; DOI : https://doi.org/10.4000/crm.5822 


\title{
酷RM
}

\section{Christine de Pizan et la répétition de l'histoire: exempla historiques et discours à caractère prophétique dans le livre I du Livre de l'advision Christine}

\begin{abstract}
In the first part of her Livre de l'Advision Cristine, Christine de Pizan uses the insertion of exempla so as to work out a prophetic discourse whose power of persuasion derives from a system of explicit and implicit analogies. Following the narratives, the exegesis tempts one to recognize in the events borrowed from the biblical texts the reflected image of the events lived by the Kingdom of France and the foreboding of its tragic fate. It links in a more complex pattern the Hebrew Kings to the French Princes, Libera's allegoric figure to the biblical prophets, and especially Christine herself to the allegory which bestows the writer the role of a prophetess. But the strength of the political discourse owns as much to this mirror effect as to the author's literary accomplishments which confer to the first book of the Advision the structure of the last evoked exemplum ${ }^{1}$.

Résumé Dans la première partie du Livre de l'Advision Cristine, Christine de Pizan se sert de l'insertion d'exempla pour élaborer un discours prophétique qui tire son pouvoir de persuasion d'un système d'analogies explicites et implicites. L'exégèse qui suit les récits invite à voir dans les faits tirés de l'histoire biblique le reflet des événements vécus par le royaume de France et l'annonce de son destin tragique. Des liens plus complexes sont instaurés entre les rois hébreux et les princes français, entre la figure allégorique de Libera et les prophètes du passé, et surtout entre Christine elle-même et l'allégorie qui accorde à l'écrivain la fonction de prophétesse. Mais la force du discours politique tient autant à ce système de miroirs qu'à l'art littéraire de l'écrivain qui donne au livre I de l'Advision la structure du dernier exemplum évoqué.
\end{abstract}

L'histoire tient une place prépondérante dans l'œuvre de Christine de Pizan. L'écrivain y a puisé la matière d'ouvrages conséquents; elle a également illustré ses textes allégoriques et moraux d'exempla tirés de faits antiques, bibliques, ou bien encore inspirés par un passé plus récent.

Pour Christine, comme pour ses contemporains, le récit historique conjugue plaisir et utilité : les références aux événements du passé doivent être riches d'enseignement. À ce titre, les exempla possèdent l'avantage de présenter en quelques phrases une situation précise, susceptible de retenir l'attention du prince à éduquer, en raison de la simplicité du récit et du caractère remarquable des faits relatés. Ils rendent concrète la leçon et montrent que la perfection morale exigée du prince est possible à atteindre dans la mesure où un gouverneur du passé l'a illustrée. Plus généralement, les exempla apportent la preuve qu'une situation, bonne

\footnotetext{
${ }^{1}$ Je remercie Sandrine Mats du soin avec lequel elle m'a aidée à élaborer ce résumé en anglais.
}

Cahiers de Recherches Médiévales, 15, 2008 
ou mauvaise, peut advenir de nouveau parce qu'elle s'est vérifiée dans le passé. Ce principe de répétition intervient dans l'argumentation mise en place par Christine dans son œuvre politique. Le prince est fortement encouragé à imiter ses prédécesseurs illustres et à égaler leur grandeur morale et politique, ou bien, au contraire, à blâmer et à fuir les exemples des mauvais gouverneurs.

L'utilisation de l'histoire pour démontrer le caractère prévisible de certains événements peut être mise en relation avec la conception aristotélicienne de l'histoire selon laquelle un événement est historique s'il se répète ${ }^{2}$. Il est possible d'en tirer un enseignement, puisque aux mêmes causes correspondent des conséquences similaires. Ce principe de répétition possède des implications dans la construction d'un discours de persuasion. Aristote conseille, au livre II de la Rhétorique, de donner des exemples historiques pour aider l'interlocuteur à prendre la bonne décision:

Il est plus facile de se procurer les arguments par les fables, tandis qu'il est plus utile pour la délibération d'argumenter par les faits réels, car le plus souvent l'avenir est semblable à l'advenu'.

Tout en se plaçant dans la tradition homilétique en usage au cours du Moyen Age qui fait des exempla un élément essentiel de l'édification des fidèles, lorsqu'elle a recours à ces éléments textuels, Christine se conforme cette recommandation d'Aristote. Elle tire une connaissance du futur de l'observation du passé. Mais, dans la civilisation chrétienne du Moyen Âge, ce savoir issu de la connaissance du mécanisme animant l'histoire humaine se teinte de prophétie : la prédiction des faits à venir ne peut venir que de Dieu et, surtout, le déterminisme que la conception aristotélicienne de l'histoire suppose devient signe de l'intervention divine dans les destinées humaines. Le discours bénéficie alors d'une autorité incontestable.

Christine de Pizan n'est pas la seule, à la fin du Moyen Age, à bâtir un discours à caractère prophétique à partir de récits courts empruntés à l'histoire. Elle fait de ce procédé une constante de ses ouvrages politiques même si cette technique est de moins en moins présente à mesure que l'œuvre se construit et que la voix de l'écrivain s'affermit. La première partie du Livre de l'advision Cristine, composé en 1405 , se situe au moment de sa carrière où Christine commence à élaborer des textes

${ }^{2}$ K. Stieler, «L'Histoire comme Exemple, l'Exemple comme Histoire : Contribution à la pragmatique et à la poétique des textes narratifs », Poétique, 1972, n 10, p. 176-198, p. 183 : «Les événements historiques, de par leur nature même, ne sont pas uniques; ils se répètent. On pourrait dire aussi, inversement : est historique au sens aristotélicien du terme non pas ce qui est unique, mais ce qui se répète. C'est ainsi que l'exemple nomme un ensemble formé par la situation et l'issue de la situation qui, par son retour constant, possède une signification générale. C'est pourquoi, selon Aristote, l'exemple possède véritablement un caractère anticipatoire, qui permet à quelqu'un de reconnaître sa propre situation, encore ouverte, à la lumière d'une expérience antérieure, et de prendre ainsi une décision fondée qui ne procède pas seulement d'un sophisme rhétorique. »

${ }^{3}$ Aristote, Rhétorique, trad. J. Lauxerois, Paris, Pocket, 2007, (Agora, 291), livre II, chapitre XX, p. 185. 
didactiques à l'usage des princes et où elle cherche à s'affirmer en tant qu'écrivain possédant un discours politique. En alliant exempla, critique politique et visée prophétique, le début de ce songe allégorique est représentatif d'une construction littéraire qui sert à imposer un discours politique.

Le livre I de l'Advision Cristine met en scène l'allégorie du royaume de France, sous la forme de Libera, la Dame Couronnée. Libera raconte son histoire à la narratrice, Christine, et se plaint très longuement des malheurs qu'elle subit. Elle tient pour responsables ses enfants et sujets, soumis aux vices, de la situation catastrophique de son royaume. Elle clôt son discours par une série de mises en gardes qui annonce l'imminence d'une punition divine. Les douze derniers chapitres du livre I sont particulièrement riches en courts récits illustratifs, dont le choix, l'insertion dans le corps du texte, la structure narrative sont soigneusement pensés dans le but de prédire aux lecteurs princiers un avenir funeste.

Dans l'ensemble de son œuvre didactique, Christine utilise en majorité des récits inspirés de l'histoire et de la mythologie grecques et latines. Or, parmi les treize exempla présents dans la fin du monologue de Libera, tous, à une exception près, sont tirés de l'Ancien Testament. $\mathrm{Si}$, en règle générale, les personnages antiques donnent des exemples à suivre, les récits vétérotestamentaires de la première partie de l'Advision Cristine répondent à un modèle très précis: le protagoniste de l'anecdote est possédé par un vice, il a la possibilité de se repentir pour éviter la colère de Dieu, mais, ne prêtant pas attention aux avertissements qui lui sont adressés, il persévère dans l'erreur et subit le châtiment qui lui était promis. Ces récits présentent donc deux constantes, essentielles dans l'élaboration du discours à caractère prophétique : leur origine et le fait raconté.

Même si la référence est parfois erronée, la source de chaque anecdote est précisée. Cette mention garantie la véracité des faits exposés, mais en plus de cautionner le contenu, elle renforce le raisonnement : la Bible fait état de cas où une attitude pécheresse a suscité la colère de Dieu ; cette situation, qui s'est produite à plusieurs reprises dans le passé, peut se reproduire. La nature sacrée de la source des récits pose la répétition de l'histoire comme un fait indéniable, et ce principe qui régit le devenir humain devient manifestation de la volonté divine.

Le simple rappel des interventions divines qui punissent la transgression des lois morales vise à susciter la peur ${ }^{4}$. Cet effet est accentué par la répétition de l'avertissement dans l'espace du texte. À partir du chapitre XXII jusqu'à la fin du livre I, chaque chapitre propose une ou deux anecdotes au contenu narratif similaire $^{5}$. Le lecteur ne peut pas échapper au message délivré : non seulement la punition divine s'est produite plusieurs fois dans l'histoire, mais le texte est saturé de la répétition de ce fait. Martelée, la promesse d'une catastrophe imminente devient oppressante et l'accumulation des exempla prend une valeur argumentative. En systématisant l'enchaînement des faits et leur conséquence, ce procédé introduit de la rationalité dans le sentiment de peur: la colère divine ne se manifeste pas au

${ }^{4}$ Avec ce procédé, Christine reste proche des conseils d'Aristote. Ibid, II, 5, p. 146-147: «Aussi bien, quand il vaut mieux que les auditeurs ressentent de la crainte, il faut les mettre dans la disposition de la ressentir, en leur disant que cela peut arriver ; car il y a des gens plus grands qu'eux à qui c'est arrivé. »

${ }^{5}$ Voir la liste des exempla en annexe. 
hasard, elle obéit à une logique selon laquelle les agissements humains jouent un rôle déterminant. Le destinataire de la leçon est ainsi renvoyé à sa propre responsabilité.

L'accumulation des exempla permet à Christine de Pizan de construire un double système analogique sur lequel repose son argumentation. La mise en parallèle de récits pris dans différents livres de l'Ancien Testament souligne la similarité des événements et pose une équivalence entre les histoires racontées. Mais l'analogie repose également sur la façon dont ils sont insérer dans le corps du texte, technique qui change au cours du livre I. Des allusions très courtes à des histoires littéraires et mythologiques sont introduites dans des structures comparatives destinées à mettre en valeur le caractère excessif des souffrances de Libera et faire du royaume de France un enfer terrestre ${ }^{6}$. Puis, les exempla forment ensuite les éléments principaux d'un discours démonstratif plus complexe, où la vision eschatologique se double d'une promesse prophétique. Dans ces cas précis, qui seront ceux qui nous intéresserons exclusivement désormais, l'environnement textuel dans lequel les anecdotes s'insèrent guide avec soin la lecture vers une interprétation unique et annonciatrice d'un malheur prochain. Les termes «advision» et «prophecie» utilisés ${ }^{7}$, principalement au début du discours prémonitoire, pour désigner les courts récits signalent à la fois leur fonction prophétique et la nécessité de les interpréter pour en connaître le sens profond. Cet avertissement ne suffit pas à garantir une bonne compréhension du message politique. Pour s'assurer de la clarté de son argumentation, Christine insiste sur les liens analogiques qui rapprochent les événements de l'histoire juive de la situation contemporaine de l'écriture.

Dans les chapitres XVII, XVIII et XIX, le parallèle est établi par les phrases qui encadrent le récit. Par exemple, au chapitre XVII, l'histoire du rêve de Nabuchodonosor est précédée d'une courte introduction qui invite à lire dans l'anecdote biblique une description des malheurs subis par la Dame Couronnée et de ceux qui risquent d'advenir :

De l'enfleure de ceste maladie qui ainsi court parmi ma terre et de l'inconvenient ou elle tire et qu'il s'en ensuivra, recite le prophete Daniel ou .IIIe. chapitre l'advision qui vid jadis l'orgueilleux roy Nabugodonozor ${ }^{8}$.

Puis l'exemplum est suivi d'un commentaire prenant en compte chaque élément du récit et le rapprochant de la situation vécue par Libera. Ainsi, l'arbre du songe fait référence aux orgueilleux du royaume de France qui, assurés de leur puissance, défient Dieu et l'Église :

\footnotetext{
${ }^{6}$ Le Livre de l'advision Cristine, éd. C. Reno et L. Dulac, Paris, Champion, 2001, I, XVI, 1. 41-43, p. 30: «Mieulx t'aduisist acompaignier Proserpine avec Megera, Alecto et Thesiphoné, deesses de rage infernale, qu'estre establie a ceste court !»;1. 93-94, p. 32 : « Si est tant commune ceste plaie, non mendre que celles d'Egipte en mon royaume »

${ }^{7}$ Voir par exemple : ibid., I, XVII, p. 36, 1. 10-11 ; I, XXVII, p. 46, 1. 35.

${ }^{8}$ Ibid., I, XVII, 1. 2-5, p. 32.
} 
Cestui dit arbre segnefie les enflez devant dis tres puissans qui sont logiez es haulz dongions de ma terre recevent le vent de perdicion, lesquelz sont de si grant estat, force et puissance qu'a pou cuident attaindre au ciel ; et tant les rent infetz l'enfleure dudit vent que non pas seulement contre les creatures leurs courages sont gros, qui raempliz de desdaing les demonstre, mais aussi contre Dieu et son Eglise?

Cette interprétation se termine par la description du châtiment subi par le roi de Babylone. L'explication détaillée du début rend inutile de s'appesantir sur l'avertissement. En n'ajoutant aucune explication, la locutrice s'éclipse pour laisser place à la seule colère divine. Ce procédé accentue l'effet dramatique déjà produit par l'analogie.

Le récit est donc enchâssé dans un cadre qui lui assigne une interprétation bien précise : les vices règnent sur le royaume de France et la colère de Dieu sera la conséquence logique de leurs méfaits. Ce cadre assure une continuité entre le discours argumentatif de la Dame Couronnée et le récit. Le passage interprétatif ne fait que renforcer les liens entre les quelques lignes narratives et le reste du monologue. D'ailleurs, il faut noter que l'interprétation donnée par Libera est presque aussi obscure que le récit biblique lui-même, de sorte qu'on peut s'interroger sur la réelle fonction de cette exégèse. Outre qu'elles témoignent de la difficulté pour Christine de Pizan de désigner clairement les puissants comme responsables des malheurs de la France, ces explications absconses sont l'occasion de développer l'effet prophétique déjà présent dans les exempla en introduisant un lexique digne des descriptions de l'apocalypse : "vent de perdicion », « infetz», «enfleure», «creatures». L'autorité de Libera en tant que porteuse d'une connaissance du futur s'en trouve renforcée.

Dans ces chapitres, le discours à caractère prophétique obéit donc à une construction bien définie qui désigne clairement les vices comme coupables des malheurs de la France mais qui obscurcit délibérément la question des responsabilités humaines. Les derniers chapitres du livre I sont marqués par un changement radical de ce principe de composition. À partir du chapitre XXII, le lien qui unit l'exemplum au reste du texte se fait plus lâche. La phrase introductive invite encore à voir dans l'histoire biblique un reflet des souffrances du royaume de France $^{10}$. Mais le commentaire qui interprétait chaque élément du récit disparaît. Le récit est immédiatement suivi par des plaintes sur les malheurs qui viendront assurément $^{11}$. Comme dans les passages étudiés précédemment, l'avertissement est formulé sans détour. Mais, en supprimant l'exégèse, Christine évite que l'identification des responsables des malheurs de la France soit trop évidente. Le

\footnotetext{
${ }^{9}$ Ibid., I, XVII, 1. 15-18, p. 32.

${ }^{10}$ Ibid., I, XXII, 1. 9-14, p. 39 : «Helas ! n'ay je cause de penser la figure de ma ruine en ce qu'il est escript ou .XV'. chappitre du Livre des Roys que, comme Dieu de loy divine repreuve ceste dicte vicieuse guespe si comme sa contraire, pour ycelle et a sa cause debouta Saul roy que elle avoit aluché lors que il l'ot envoié en bataille contre le roy de Amalec?» ${ }^{11}$ Ibid., I, XXII, 1. 29-31, p. 39 : «Ha ! chiere amie, et dois je penser que Dieu dorme ? Ne voy je le temps que contre ses commandemens sont espargniez ses justices sur les mauvais, de droit divin condampnez a punicion?»
} 
commentaire du rêve de Nabuchodonosor laissait entrevoir les princes derrière l'expression «tres puissans qui sont logiez es haulz dongions de [la] terre [de Libera]». Par la suite, en ne posant pas de parallèle explicite avec la situation du royaume en conclusion de l'exemplum, et, de ce fait, en liant moins fortement le récit au texte qui l'accueille, Christine semble déplacer son intérêt de la désignation des responsables sur la menace de punition et accentue l'effet dramatique de la fin du récit. Au discours rationnel de l'exégèse, semble se substituer un discours qui suscite l'émotion en mettant l'accent sur les châtiments à venir et sur la tristesse de la Dame Couronnée.

Cependant, une lecture détaillée des anecdotes dément l'impression première d'une condamnation certes virulente mais dont l'imprécision nuit à la vigueur. L'analogie explicite de l'introduction se double d'une autre plus discrète qui repose sur les éléments narratifs et qui donne lieu à une critique plus précise. La fonction des personnages principaux, soigneusement mentionnée, circonscrit le groupe incriminé : tous les récits mettent en scène un roi. Les histoires de Saül et de Balthasar montrent des souverains agissant sous l'emprise de l'avarice, celles de David et de Nabuchodonosor témoignent des méfaits de l'orgueil sur les puissants. Enfin, l'anecdote concernant Sédécias condamne le manque de loyauté de ce gouverneur. Or, les personnages mis en scène sont tellement connus que Christine aurait pu s'abstenir de rappeler leur fonction dans des récits qui se limitent aux éléments narratifs essentiels à la compréhension de l'histoire. Sa volonté d'insister sur leur statut de gouverneur est manifeste. Les reproches de Libera s'adressent ainsi plus particulièrement aux princes de sang. Un parallèle implicite, fondé sur le processus d'identification entre le lecteur et le personnage principal de l'anecdote, est créé qui donne aux critiques générales et morales des phrases introductives une portée plus politique.

Mais le système analogique n'est pas clos sur lui-même puisque le lecteur est invité à s'inscrire dans la série des anecdotes et à contempler dans les situations et dans les personnages exposés une représentation de son propre devenir. Au-delà de la prédiction d'un futur funeste, la mise en scène de personnages habités de vices constitue une critique sévère des destinataires du discours véhément de Libera. Grâce à l'accumulation des exempla, la Dame Couronnée tend au lecteur un miroir composé de plusieurs surfaces réfléchissantes, renvoyant toutes la même image dont la multiplication à l'identique garantit l'exactitude et oblige à accepter la réalité déplaisante rendue ainsi visible. S'il est menaçant, le discours prophétique est aussi et avant tout dénonciation d'un comportement et invitation à prendre conscience du fourvoiement auquel il conduit.

Les conclusions des récits confirment l'orientation politique donnée par la présentation extrêmement limitée des personnages principaux. Après avoir décrit brièvement les faits condamnables des souverains, Christine s'appesantit sur les manifestations de la colère divine: les tortures physiques et morales subies par les pécheurs sont mentionnées, les paroles de Dieu annonçant les punitions sont citées. Mais ces descriptions, parfois complaisantes ${ }^{12}$, des punitions divines

${ }^{12}$ Voir par exemple, ibid., I, XXVI, p. 45, 1. 24-26 : «Et adonc devant [Zedechias] occirre fist ses enfans et a lui crever les deux yeulx, et lié de chaiennes le fist prisonnier mener en Babiloine. » 
s'accompagnent de la mention systématique d'un autre châtiment : la perte du pouvoir royal ${ }^{13}$. Placées en conclusion des récits, les conséquences politiques apparaissent comme l'étape ultime de la punition divine et à ce titre, elles semblent plus menaçantes que les tortures personnelles subies par le souverain.

De plus, bien souvent, la déchéance royale se double de la destruction du royaume. Ainsi, au chapitre XXIV, l'orgueil de David est puni par la mort d'une partie de son peuple ${ }^{14}$; au chapitre XXVII, le royaume de Balthasar est divisé et livré à deux rois étrangers ${ }^{15}$. Christine rappelle ainsi les responsabilités du prince à l'égard de son peuple: s'il se conduit mal, non seulement il risque de voir le pouvoir lui échapper, mais il met son royaume en danger et fait souffrir ses sujets. Ces conclusions associent définitivement la condamnation des péchés à la question du bon ou du mauvais gouvernement. En jouant sur le dit et le non-dit, l'auteur multiplie les effets narratifs et sa critique des puissants, difficile à prononcer sans voile, est malgré tout compréhensible pour qui prend la peine de déchiffrer le récit.

L'élaboration d'une critique politique se fait donc en deux temps: tout d'abord la mise en place d'un système de lecture attire l'attention du lecteur sur le fait que chaque détail de l'anecdote peut être mis en relation avec la situation contemporaine du royaume de France; puis une légère distension de ce système analogique recentre le parallèle sur les rapports entre le prince hébreu et le prince français, lecteur potentiel de l'Advision Cristine. Cette seconde phase est rendue possible par l'existence de la première. Plusieurs éléments appartenant à la technique de la compilation interviennent donc dans l'élaboration du discours prophétique et politique: principe d'accumulation des exempla de même type qui souligne la répétition de l'histoire et le caractère implacable de son mécanisme ; insertion d'un élément disparate dans le texte auquel est attribué une fonction bien précise le reliant par plusieurs points au discours dans lequel il prend place; principe d'identification qui poursuit l'analogie hors du texte et atteint le lecteur en lui rappelant sa fonction sociale et en lui présentant un miroir peu flatteur.

Ces chapitres de l'Advision Cristine délivrent un message bien sombre. Pourtant, le discours de Libera ne s'enferme pas dans ce pessimisme. Le processus d'identification et sa complexification dans l'avant-dernier chapitre du livre I proposent une alternative au chaos. En opposition aux exempla qui promettent un avenir funeste aux seigneurs, les deux derniers récits proposent une possibilité d'échapper à la sentence divine. Juste avant de conclure sa plainte, la Dame Couronnée évoque les histoires de Cassandre et du roi de Ninive. Ces deux récits comportent les mêmes détails que les anecdotes précédentes : des gouverneurs sont confrontés à l'erreur ou au péché ; ils reçoivent les avertissements d'une déchéance prochaine s'ils poursuivent dans cette voie; selon qu'ils persévèrent dans le même comportement ou qu'ils se repentent, les pécheurs subissent ou non la punition divine. Les phases des récits sont donc toujours similaires, mais avec l'anecdote du roi de Ninive, Christine introduit une variante qui rompt l'accumulation de récits strictement identiques et qui montre que le mécanisme implacable de l'histoire se

${ }^{13}$ Ibid., I, XXII, 1. 25-28, p. 39 ; I, XXVII, 1. 54-59, p. 47.

${ }^{14}$ Ibid., I, XXIV, p. 42.

${ }^{15}$ Ibid., I, XXVII, p. 47. 
modifie lorsqu'un élément de la chaîne factuelle est changé. Le cours des événements peut être influencé par la décision humaine d'un gouverneur éclairé qui prend conscience de ses erreurs et choisit la contrition plutôt que la destruction de son peuple. La juxtaposition des deux anecdotes permet au lecteur princier d'expérimenter par procuration deux situations différentes et d'agir en connaissance de cause. Son choix est bien sûr influencé par les avis émis par Libera sur les deux conduites possibles: l'anecdote de Cassandre est introduite par les plaintes de la Dame Couronnée alors que l'attitude du roi de Ninive est valorisée par la louange de la sagesse du souverain: "O saige roy de Ninive bien conseillez ${ }^{16}$. Cette interpellation, qui introduit un discours direct, contraint le lecteur à se mettre à la place du monarque biblique. Il y est également encouragé par la présentation du personnage, défini comme un individu précis, possédant un nom et une fonction, alors qu'il est beaucoup plus difficile de s'identifier au groupe informe des Troyens ${ }^{17}$. De plus, le choix d'un récit troyen et d'un récit biblique n'est pas innocent. Christine joue sur la double origine mythique de la France et de ses gouverneurs. La dynastie des rois de France se conçoit comme l'héritière directe de la famille royale troyenne et elle exerce son pouvoir sur le nouveau peuple élu de Dieu. Les lecteurs ne peuvent voir dans ces deux exempla qu'un encouragement supplémentaire à s'identifier aux personnages qu'ils mettent en scène. En indiquant sa préférence pour le roi hébreu sur le peuple troyen, Christine préconise de suivre la voie de la morale plutôt que celle de la guerre. Surtout, en valorisant l'attitude du roi de Ninive, elle rappelle la fonction essentielle du souverain qui doit être le premier à se repentir et qui doit imposer à son peuple de l'imiter. Il est responsable, par son attitude, des péchés de ses sujets et il a pour devoir d'y remédier.

$\mathrm{Si}$, dans ces anecdotes, le parallèle entre le protagoniste et le lecteur est maintes fois suggéré, d'autres analogies sont mises en évidence. Les deux récits attribuent une place essentielle au prophète : Cassandre et Jonas tentent d'infléchir le comportement des pécheurs. Le personnage du prophète est certes déjà présent dans certains des exempla précédents, mais il possède alors un rôle différent. Dieu charge Samuel de destituer David ${ }^{18}$ et il ordonne au même roi de choisir entre trois châtiments par l'intermédiaire de $\mathrm{Gad}^{19}$. Quand il s'agit de menacer les princes, le prophète est avant tout chargé d'exécuter la punition. Dans l'anecdote de Cassandre et, dans une moindre mesure, dans celle du roi de Ninive, ce personnage n'est plus un simple exécutant de la colère de Dieu. Il avertit le prince de la menace qui pèse sur lui ; il l'encourage à reconnaître ses erreurs et à se repentir. Il est donc l'élément déclencheur qui permet une prise de conscience et un changement de comportement.

Alliant une nouvelle fois la comparaison et l'analogie implicite, Christine de Pizan procède à une double identification à partir du personnage du prophète. L'épisode de Cassandre installe très clairement Libera dans le rôle de la prophétesse dès la phrase qui introduit le récit :

\footnotetext{
${ }^{16}$ Ibid., I, XXVIII, 1. 38, p. 48.

${ }^{17}$ Ibid., I, XXVIII, 1. 30, p. 48 : « Cassandra, la saige filled u roy Priant, qui, voiant la ruine apparaillee sus les Troyens $»$.

${ }^{18}$ Ibid., I, XXII, 1. 19-28, p. 39.

${ }^{19}$ Ibid., I, XXIV, 1. 10-15, p. 42.
} 
Helas! j'ay grant paour que semblable je soie a Cassandra, la saige fille du roy Priant, qui, voiant la ruine appareillee sus les Troyens, les amonnestoit d'appaisier leurs couraiges contre Grigois, ains que pis leur venist ${ }^{20}$.

Le rapprochement est aisé, l'identité féminine faisant le lien avec le personnage antique. Pourtant Christine aurait pu aussi établir un parallèle plus marqué entre la Dame Couronnée et Jonas, le prophète qui a été écouté. L'identification est juste suggérée par la comparaison explicite de l'anecdote troyenne et de la situation du royaume. Les mentions d'autres prophètes dans les chapitres précédents tissent également le lien analogique: Samuel, Gad, Merlin, Joachim de Flore et les Sibylles. En mentionnant leur rôle d'intermédiaire entre Dieu et les hommes, Libera se revendique de leur sagesse. De même, lorsque les paroles de Dieu ou celles des prophètes sont citées au style direct, l'allégorie se fait porteuse du message divin ${ }^{21}$.

Toutefois, l'absence de parallèle parfaitement lisible avec un prophète biblique est à interroger. La volonté de trouver dans l'histoire un précédent de prophète femme entre sans doute en jeu, de même que le désir de dramatiser son discours en lui superposant celui de la mère déchirée. Il semble surtout que Christine ait choisi délibérément une figure de prophète non pas triomphante mais bien au contraire désespérée à la fois de l'état politique du royaume et de son incapacité de se faire entendre par les puissants. Dans le Livre de la cité des dames, composé à peu près à la même date, Christine développe le désespoir de Cassandre, causé par la connaissance d'un avenir tourmenté et par l'incapacité de ses mises en garde à atteindre la conscience des gouverneurs de Troie ${ }^{22}$. De plus, l'association des larmes à la clairvoyance prophétique constitue une tradition néotestamentaire, dont Christine peut s'inspirer en la déplaçant sur un personnage antique: Jésus Christ pleure au jardin des Oliviers à la pensée de la Passion; dans l'Apocalypse, saint Jean pleure de connaitre un futur effroyable ${ }^{23}$.

${ }^{20}$ Ibid., I, XXVIII, 1. 28-31, p. 48.

${ }^{21}$ Ibid., I, XXII, 1. 25-27, p. 39 ; I, XXIV, 11. 24-27, p. 42 ; XXVI, 11. 23-24, p. 45.

${ }^{22}$ La Cité des dames, trad. T. Moreau et E. Hicks, Paris, Stock, 1986, (Moyen Age), p. 135 : «Dans toute la période qui précéda cette boucherie entre Grecs et Troyens, plus elle voyait fleurir les fortunes de Troie et sa magnificence, plus elle pleurait, se lamentant et menant grand deuil. (...) Lorsqu'elle vit éclater la guerre, ses pleurs redoublèrent ; elle criait, pleurait, harcelait sans cesse son père et ses frères, leur demandant pour l'amour du ciel de faire la paix avec les Grecs, car sinon cette guerre les détruirait lamentablement. Toutefois, comme elle ne cessait de se lamenter - et à juste titre, car elle ne pouvait taire un tel désastre et tant de malheurs -, son père et ses frères la battirent à maintes reprises, disant qu'elle était folle. »

${ }^{23} \mathrm{P}$. Nagy, Le Don des larmes au Moyen Âge : Un instrument spirituel en quête d'institution ( $V^{e}-X I I I^{e}$ siècle), Paris, Albin Michel, 2000, p. 46-48. Sur les pleurs de Jésus Christ, p. 46 : « Ils dessinent le modèle chrétien de la valorisation de l'expression des sentiments, tout en présentant une particularité bien comprise et exploité par le christianisme: celle de relier l'émotion subite à l'avenir pressenti. Cette articulation entre émotions humaines et savoir surhumain, qui se retrouve dans les pleurs de l'Apocalypse, organise l'exégèse patristique et médiévale des passages concernés. » 
Les 'enfants' de la Dame Couronnée sont ainsi accusés de manque de discernement. La menace de guerre est clairement formulée, et renvoie aux risques de guerre civile qui conduiraient à la destruction du royaume de France ${ }^{24}$. La figure de Libera et son discours sont alors les porte-parole de la pensée politique de Christine, inquiète du chaos qui menace la France et de ne pas être entendue par les princes, elle qui est aussi une femme mise à mal par les vicissitudes de l'existence, comme elle le rappellera dans le livre III, et qui cherche à imposer sa sagesse dans un monde dominé par les intérêts personnels des gouverneurs.

Il convient également de mettre le choix de Cassandre sur le compte d'une modestie, vite démentie mais essentielle pour l'introduire l'idée d'une possibilité pour l'homme d'agir sur l'histoire. En identifiant Libera à une femme païenne, Christine insiste sur son humanité, oubliant un instant que la Dame Couronnée est une allégorie issue du monde divin des idées. Mais cette humilité n'est en fait qu'un moyen de rappeler la nature cléricale de sa connaissance. Au cours de son discours, Libera multiplie les marques d'érudition: elle glisse des citations latines dans sa description des méfaits des vices ${ }^{25}$; elle mentionne les références des exempla ; elle développe une interprétation exégétique qu'elle fonde, par endroits, sur l'étymologie ${ }^{26}$. Derrière le récit apocalyptique des souffrances du royaume et les menaces d'une intervention surnaturelle, se profile un discours plus rationnel, qui, fondé sur une maîtrise des outils intellectuels des clercs, s'adresse à l'intelligence des princes. Par cet appel à la raison, Christine demande aux gouverneurs de se conduire en êtres humains. Le bestiaire ${ }^{27}$ des chapitres précédents les associe à des animaux dangereux ${ }^{28}$. Ils doivent désormais égaler le seul monarque qui garde sa forme humaine dans le texte : Charles V, le bon époux du royaume de France, le seul jardinier qui a su faire prospérer ses terres à la perfection, le roi idéal ${ }^{29}$ qui utilise sa sagesse plutôt que la violence. Le second enjeu de cette démonstration d'érudition est de prouver que le savoir humain peut avoir une incidence sur le cours de l'histoire. Pour cela, les gouverneurs doivent suivre les conseils avisés des sages lettrés qui les entourent, dont, précisément, l'écrivain.

Car au creux du portrait de Libera en clergesse se dessine celui de Christine de Pizan elle-même. Selon le même processus qui encourage les princes à se percevoir sous les traits d'un souverain biblique, une analogie implicite se tisse entre l'allégorie et l'écrivain, dont les points d'appui se forment à partir des

\footnotetext{
${ }^{24}$ L'Advision Cristine, op. cit., I, XXVIII, 1. 34-35, p. 48 : « Ainsi de eulx amender et mettre a paix devers leur Dieu de qui ja voy la guerre, bien vouldroye qu'a mes paroles si adjoustassent foy ».

${ }^{25}$ Voir par exemple, ibid., I, XVII, p. 33.

${ }^{26}$ Ibid., I, XX, 1. 12-13, p. 36.

${ }^{27}$ L. Dulac, «Sur les fonctions du bestiaire dans quelques œuvres didactiques de Christine de Pizan », «Riens ne m'est seur que la chose incertaine » : Études sur l'art d'écrire au Moyen Âge offertes à Eric Hicks par ses anciens élèves et ses amis, Paris, Honoré Champion, 2001, p. 181-194.

${ }^{28}$ Voir par exemple le chapitre consacré à Charles VI et à Louis d'Orléans, où le roi et son frère sont comparés à des oiseaux de proie, certes beaux et domestiqués mais capables de donner la mort. Ibid., I, XII, p. 23-24.

${ }^{29}$ Ibid., I, p. 21-23.
} 
caractéristiques communes : la féminité, l'humanité, une existence tourmentée et marquée par le veuvage, la revendication d'une culture cléricale, la maîtrise du discours. Il ne s'agit pas d'affirmer que Christine de Pizan s'identifie à la France dans cette première partie de l'Advision Cristine. Elle accomplit déjà une avancée dans l'histoire des idées politiques en donnant une forme cohérente et aboutie à toutes les tentatives d'allégorisation du royaume réalisées avant elle ${ }^{30}$. Il est sans doute inconcevable pour elle de se projeter dans la personnification d'une entité encore mal définie, même si elle se sert parfois d'un discours féminin déjà élaboré dans sa poésie lyrique pour bâtir cette figure inédite ${ }^{31}$. Cependant, il est indéniable que Christine utilise quelques traits du portrait de Libera pour asseoir sa propre autorité d'écrivain. En montrant que les propos de la Dame Couronnée méritent d'être écoutés, l'écrivain valorise la sagesse de son propre discours et espère que ses lecteurs y prêteront attention.

De même, la possession par Libera d'un savoir prophétique, à la fois basé sur la connaissance de l'histoire et sur la croyance profonde en l'intrusion de Dieu dans le devenir des hommes, contribue à construire la figure de l'écrivain en prophétesse. Cette figure se retrouve dans nombre des œuvres politiques de Christine de Pizan et le livre I de l'Advision Cristine témoigne d'une des premières tentatives de l'écrivain de constituer son autorité autour de cette image de personnage clairvoyant. Dans l'Advision Cristine, la figure de l'écrivain prophète apparaît avec prudence, presque avec timidité, endossée par une allégorie dont Christine se rapproche. Pourtant, on trouve dans ce traité un élément essentiel de l'élaboration du discours à caractère prophétique : le récit du repentir du roi de Ninive et surtout, l'imitation de sa construction narrative. Jean-Claude Mühlethaler a montré l'influence de cet épisode biblique sur la structure de la Lamentacion sur les maux de la France et sur l'émergence d'une voix prophétique ${ }^{32}$. Cinq ans avant la composition de ce texte, le discours de Libera imite déjà les trois phases de ce récit. La Dame Couronnée constate et dénonce la dépravation du peuple français, puis elle annonce le danger

${ }^{30}$ T. Lassabatère, «La personnification de la France dans la littérature de la fin du Moyen Âge. Autour d'Eustache Deschamps et Christine de Pizan ", Contexts and continuities: Proceedings of the IVth International Colloquium on Christine de Pizan (Glasgow 21-27 July 2000), dir. A. Kennedy, Glasgow, University of Glasgow Press, 2002, p. 483-504.

${ }^{31}$ Elle reprend par exemple la figure de la veuve éplorée. Voir C. Le Ninan, Le Sage roi et la "clergesse » : L'écriture du politique dans l'œuvre de Christine de Pizan, thèse de doctorat rédigée sous la direction d'E. Baumgartner, Paris III, 2004, chap. I, p. 62-73.

${ }^{32}$ J.-C. Mühlethaler, «Le Poète et le prophète : Littérature et politique au $\mathrm{XV}^{\mathrm{e}}$ siècle », Le Moyen Français, 1983, n 13 , p. 38-39: «Du troisième chapitre du livre de Jonas, la Lamentacion retient trois éléments-clés correspondant aux trois phases du récit biblique: l'iniquité du peuple, la colère divine et la punition détournée par le repentir et la prière. C'est là une grille que la narratrice utilise pour analyser la réalité contemporaine; la situation actuelle s'identifie à la deuxième phase du récit biblique, la troisième phase restant pour le moment au stade virtuel. Il incombe à la narratrice de la Lamentacion de la faire réaliser par les narrataires, leur proposant un programme d'action que Dieu valorisera positivement comme il a valorisé positivement le programme de repentir imposé par le roi aux Ninivites. Cette orientation du discours en fait, de prime abord, un discours prophétique ; la narratrice y fait figure de prophète de l'Ancien Testament. » 
d'une colère divine et énonce la possibilité d'un repentir qui écarterait définitivement tout risque de punition. En faisant correspondre la structure du discours de l'allégorie à celle du récit biblique, Christine s'inscrit parmi les prophètes chargés de délivrer le message de Dieu qui changera le destin du royaume. L'insertion de l'exemplum du roi de Ninive à la fin du livre I est une invitation à relire les chapitres précédents à la lumière du récit d'un événement que l'écrivain souhaite voir se reproduire.

C'est donc à l'écrivain que revient la fonction d'avertir et de convaincre ses contemporains, et pour y parvenir, Christine s'appuie autant sur son adresse à organiser solidement ses arguments que sur sa capacité, purement littéraire, d'imiter la structure d'un récit et de la détacher de son contexte premier pour l'adapter à une autre situation qu'elle servira à analyser et qu'elle contribuera, dans le meilleur des cas, à améliorer. Cet art littéraire, qui utilise largement, comme nous l'avons vu, les ressorts de la narration dans l'espace textuel très réduit des exempla - les effets dramatiques, l'identification, l'organisation du récit -, fait l'intérêt et la qualité des premières œuvres politique de l'écrivain. Il se fera plus rare dans les œuvres plus tardives, en particulier dans le Livre de paix, où la volonté de Christine de se mesurer aux philosophes antiques les plus renommés la conduira à réduire les exempla à de simples allusions, comme elle le fait déjà, dans l'Advision, lorsqu'au chapitre consacré à la luxure, les références historiques s'amoncellent sans bénéficier d'aucun développement ${ }^{33}$. L'habileté de l'écrivain transparait alors dans la réécriture des citations philosophiques grâce auxquels elle cherche sa place parmi les grands penseurs.

Dans l'Advision Cristine, le discours politique puise sa force dans la fragilité de la voix de l'écrivain qui, entre humilité et affirmation, donne les preuves des limites de sa connaissance du futur. Le choix laissé aux lecteurs de se conformer à l'un ou l'autre des comportements décrits par les deux derniers exempla montre que Christine est capable de prévoir ce que serait l'avenir dans des cas précis, mais qu'elle est incapable de prédire avec certitude quel sera le destin de la France. De même, en se contentant d'accusations imprécises et en refusant de se prononcer clairement dans la lutte pour le pouvoir qui déchire les princes, Christine montre à quel point il est difficile pour un écrivain vivant de sa plume de prendre parti. Les solutions qu'elle propose pour sauver le royaume se fondent sur des idées théoriques qui exigent des gouverneurs sagesse, vertu et souci du bien commun. Contemporaine du Livre des fais et bonnes meurs du sage roy Charles V, l'Advision Cristine porte les traces des recherches de l'écrivain pour concilier pratique du pouvoir et idéal politique. Le traité allégorique peut être perçu comme l'envers réaliste de la biographie royale: alors que la vie de Charles $\mathrm{V}$ est en parfaite cohérence avec les principes du bon gouvernement, les princes malfaisants de l'Advision s'illustrent par leur incapacité à diriger le royaume.

Claire Le Ninan

CEMA Paris III La Sorbonne Nouvelle

${ }^{33}$ Advision Cristine, op. cit., I, XXV, 1. 11-25, p. 43. 


\section{Annexe}

Liste des exempla du discours prophétique du livre I du Livre de l'advision Cristine.

I, XVII, 1. 2-14, p. 32 : le songe de Nabuchodonosor

I, XVIII, 1. 9-19, p. 33-34 : le songe de Zacharie

I, XX, 1. 10-23, p. 36-37 : Samson et les renards

I, XXII, 1. 9-28, p. 39 : la destitution de Saul.

I, XXIII, 1. 8-14, p. 41 : Le peuple d'Israël

I, XXIV, 1. 5-15, p. 42 : la punition de David

I, XXIV, 1. 17-30, p. 42 : Nabuchodonosor transformé en bête

I, XXV, 1. 39-41, p. 44 : Noé et le déluge

I, XXVI, 1. 18-26, p. 45 : Sédécias

I, XXVII, 1. 12-19, p. 46 : Caïn

I, XXVII, 1. 25- 59, p. 46-47 : Balthasar

I, XXVIII, 1. 28-33, p. 48 : Cassandre

I, XXVIII, 1. 38-45, p. 48 : Le roi de Ninive et Jonas 